\title{
An underrated variable essential for tailoring the structure of xerogel: the methanol content of commercial formaldehyde solutions
}

Isabel D. Alonso-Buenaposada ${ }^{1}$, Leoncio Garrido ${ }^{2}$, M.A. Montes-Morán ${ }^{1}, J_{\text {. Angel Menéndez }}{ }^{1}$, Ana Arenillas ${ }^{1 *}$

${ }^{1}$ Instituto Nacional del Carbón, CSIC, Apartado 73, 33080 Oviedo, Spain

${ }^{2}$ Departamento de Química Física, Instituto de Ciencia y Tecnología de Polímeros, ICTP-CSIC, Juan de la Cierva 3, 28006 Madrid, Spain

*Corresponding author: A. Arenillas, INCAR-CSIC, Apartado 7333080 Oviedo SPAIN, aapuente@incar.csic.es, Tel.: +34 985119090, Fax: +34 985297662

KEYWORDS: xerogel, formaldehyde, methanol, controlled porosity.

\section{ABSTRACT}

Resorcinol-formaldehyde xerogels are polymers whose porosity can be designed for a specific application by the selection of appropriate physical and chemical synthesis variables. Until recently, the methanol content of commercial formaldehyde solutions has never been considered as a chemical variable that must also be taken into account. However, it has been demonstrated that the proportion of methanol might be even more important than other variables for tailoring the porosity of xerogels.

Different reaction mechanisms are proposed to explain this heavy dependence of the final structure of RF xerogels. Organic and carbon xerogels synthesized with low concentrations of methanol showed a higher porosity with much larger pore sizes (up to two orders of magnitude) than when using formaldehyde with high concentrations of methanol. This means that extra caution needs to be shown in choosing these commercial products since formaldehyde solutions differ greatly in methanol content from one supplier to another. 



\section{Introduction}

Porous carbon and organic gels synthesized by means of Pekala's method [1], with resorcinol and formaldehyde as precursors, and water as solvent [2-5], have been the most common type of gels studied until now in preference to other precursors and reaction media [6-11].

Microwave radiation is the most common method used to synthesize this type of material since it is a highly competitive process that requires only $3 \mathrm{~h}$ instead of the 5 days needed for conventional heating. Furthermore, the entire process, from gelation and ageing to the final drying step, is carried out in the same device (referred to as one-pot synthesis), reducing the amount of energy needed and, therefore, making the process much more cost-effective.

However, the main attraction of these materials resides in their great versatility. Xerogels are considered materials with a high added value due to the fact that their porosity can be controlled and designed for a specific application by selecting appropriate values for the synthesis variables, whether physical (e.g. temperature, time of synthesis, etc.) $[2,12]$ or chemical (e.g. $\mathrm{pH}$, dilution, molar ratio resorcinol/formaldehyde, etc) [13-16]. In addition, all these variables are interdependent and an exhaustive study of all of them together requires the use of statistical programs $[13,14,17]$.

Until recently, the role of the methanol content in commercial formaldehyde solutions as a chemical variable has been underestimated. Every commercial formaldehyde solution contains a certain proportion of methanol to prevent the formaldehyde from polymerizing and precipitating, and to keep its concentration constant. It has been demonstrated [17] that the proportion of methanol is even more important than other variables for controlling porosity. As formaldehyde solutions differ greatly in methanol content from one supplier to another, this factor must be paid due attention. However, more deeply characterization of the samples obtained only varying the composition of the formaldehyde solution is needed in order to postulate a mechanism and a chemical explanation of what it is occurring.

In this work two different organic and carbon xerogels were synthesized under the same synthesis conditions using formaldehyde solutions with different proportions of methanol. A series of chemical, structural and morphological analyses of the samples obtained were carried out in order to clarify the role of the methanol content. On the basis of the results obtained, a reaction mechanism and chemical explanation for the influence of this chemical variable is proposed.

\section{Experimental}

\subsection{Synthesis of carbon xerogels}


Xerogels were synthesized by the polymerization of resorcinol (R) and formaldehyde (F) with water as the reaction media using a solution of $\mathrm{NaOH}$ to adjust the $\mathrm{pH}$ to the desired value. The variables selected for this study were: a $\mathrm{pH}$ of 5 , a dilution ratio of 8 (dilution ratio is defined as the molar ratio between the total solvent and reactants. Total solvent refers to the water and methanol contained in the formaldehyde solution and the deionized water that is added and reactants refers to the resorcinol and formaldehyde), and a R/F molar ratio of 0.7 .

In order to prepare the organic xerogels, resorcinol (Indspec, $99.6 \mathrm{wt} . \%$ ) was dissolved in deionized water in an unsealed glass beaker under magnetic stirring. As the formaldehyde solution used (Química S.A.U., 37 wt. \% formaldehyde, 0.7 wt. \% methanol, 62.3 wt. \% water) contains 0.7 wt. \% methanol, one of the gels was prepared using this solution directly. The other gel, was prepared by adding methanol (AnalaR Normapur, $99.9 \%$ ) to the former formaldehyde solution until a $12.5 \mathrm{wt}$ \% methanol content was obtained. In both cases the formaldehyde solution was then added to the rest of the precursor mixture. It is worth noting that pure methanol was added to the above formaldehyde solution, instead of using other commercial formaldehyde, in order to use exactly the same reagents for the synthesis of the samples studied. Therefore other factors such as impurities, $\mathrm{pH}$, etc. may be avoided. In order to obtain a $\mathrm{pH}$ of 5 in the precursor mixture, $5 \mathrm{M}$ and $1 \mathrm{M}$ solutions were prepared from solid $\mathrm{NaOH}$ (AnalaR Normapur, $99.9 \%$ ) and added until the desired $\mathrm{pH}$.

Each precursor solution was kept in an in-lab modified multimode microwave oven at $85{ }^{\circ} \mathrm{C}$ for the entire synthesis process (polymerization and curing steps) i.e., ca. 3 hours. The microwave cavity and the operating conditions used are described in detail elsewhere $[13,14,18]$. The polymer was then dried in the same device, by microwave heating, without any pretreatment or intermediate step until a mass loss of over $50 \mathrm{wt}$. \% was reached, giving rise to the final organic xerogel. The xerogel was then carbonized in a tubular reactor (Carbolite MTF 12/38/400) for $2 \mathrm{~h}$ at $700^{\circ} \mathrm{C}$ under a $\mathrm{N}_{2}$ flow $(150 \mathrm{~mL}$ $\min ^{-1}$ ) to obtain the carbon gel product. Samples, either organic and carbon xerogels were obtained in duplicate to ensure reproducibility.

In this study, the samples labelled OX refer to the organic xerogels, CX samples to the carbon xerogels and 0.7 or 12.5 to the percentage of methanol contained in the formaldehyde solution used in each case.

\subsection{Sample characterization}

\subsubsection{Porosity}

Before characterization, the xerogels were outgassed (Micromeritics VAcPrep 0.61) at 0.1 mbar and 120 ${ }^{\circ} \mathrm{C}$ overnight in order to remove all humidity and other physisorbed gases. 
The porosity of the samples studied was characterized by means of mercury porosimetry on an AutoPore IV 9500 (Micromeritics) device, which is able to measure from atmospheric pressure up to $228 \mathrm{MPa}$. To characterize mesoporosity the lowest limit of the apparatus considered was $5.5 \mathrm{~nm}$. Likewise, Vmacro refers to porosity ranging from 50 to $10000 \mathrm{~nm}$. The surface tension and contact angle were $485 \mathrm{mN} \mathrm{m}^{-1}$ and $130^{\circ}$, respectively, while the stem volume was between $45-58 \%$ in all the analyses performed. Initially, in the low pressure step, the samples were evacuated at $6.7 \mathrm{~Pa}$ and the equilibration time chosen was 10 seconds. Subsequently, the pressure was gradually increased to its maximum value, after which the mercury intrusion was evaluated. This characterization yielded data about pore size distribution, pore volume and bulk density.

\subsubsection{Microscopy}

Micrographs of the carbon xerogels were obtained on a Zeiss DSM 942 scanning electron microscope (SEM). For this characterization the samples were previously dried overnight and then attached to an aluminum tap using a conductive double-sided adhesive tape. A voltage of $25 \mathrm{kV}$ and a secondary electron detector EDT Everhart-Thornley, were used in all the analyses.

Transmission electron microscopy (TEM) was carried out on a Jeol 2000 at $180 \mathrm{kV}$. A very dilute ethanolic suspension was prepared and then deposited on a 200 mesh copper grid with carbon membrane.

\subsubsection{X-ray photoelectron spectroscopy (XPS)}

The X-ray photoelectron spectra (XPS) of the carbonised xerogels (CX samples) were carried out in a SPECS Phoibos 100 analyser using MgK $\alpha$ X-rays $(1254.6 \mathrm{eV})$ at a power of $100 \mathrm{~W}$ and in a residual vacuum of 10-7 Pa. Analyser pass energy of $50 \mathrm{eV}$ has been used to collect broad scan spectra (0-1100 $\mathrm{eV}$ ). The atomic percentages (atom\%) of the different elements present in the approx. $10 \mathrm{~nm}$ upper layer probed by XPS were calculated from the survey spectra by considering the integrated areas of the main XPS peaks. High resolution spectra of C (1s) were performed using a pass energy of $10 \mathrm{eV}$. C1s peaks were deconvoluted fitting Gaussian-Lorentzian functions by means of Marquardt algorithm by using proprietary software. The number of peaks considered in the fitting procedure was, on one hand, sufficient to attain a sound fitting [19], and on the other, to account for the different contributions with physical meaning. The assignment of such contribution was done following previous studies in carbon materials [20-21]. Samples were dried overnight before characterization.

\subsubsection{Nuclear Magnetic Resonance (NMR)}


The samples were analyzed directly after synthesis and also after being dried overnight under vacuum at room temperature by ${ }^{1} \mathrm{H}$ and ${ }^{13} \mathrm{C}$ solid state NMR spectroscopy and ${ }^{1} \mathrm{H}$ NMR imaging (MRI).

The ${ }^{1} \mathrm{H}$ and ${ }^{13} \mathrm{C}$ NMR measurements were performed on a Bruker Avance 400 spectrometer (Bruker BioSpin, Rheinstetten, Germany) equipped with a $89 \mathrm{~mm}$ wide bore, $9.4 \mathrm{~T}$ superconducting magnet (at ${ }^{1} \mathrm{H}$ and ${ }^{13} \mathrm{C}$ Larmor frequencies of 400.14 and $100.61 \mathrm{MHz}$, respectively). All the reported data were obtained at room temperature. Powdered samples were placed in $4 \mathrm{~mm}$ zirconia rotors and a standard Bruker double resonance $4 \mathrm{~mm}$ cross-polarization (CP)/magic angle spinning (MAS) NMR probe head was used. The ${ }^{1} \mathrm{H}$ MAS spectra were recorded using single pulse excitation and the ${ }^{13} \mathrm{C} \mathrm{CP} / \mathrm{MAS}$ spectra were obtained by applying a $3 \mathrm{~ms} \mathrm{CP}$ contact time and sideband suppression (seltics). The recycle delays in the ${ }^{1} \mathrm{H}$ and ${ }^{13} \mathrm{C}$ experiments were 5 and $3 \mathrm{~s}$, respectively. The MAS spinning rates varied between 5.0 and $7.0 \mathrm{kHz} .{ }^{13} \mathrm{C}$ NMR spectra were obtained by high-power proton decoupling at $75 \mathrm{kHz}$.

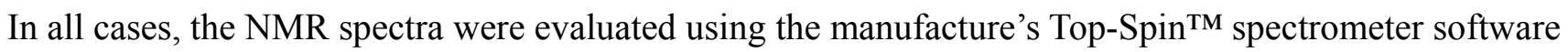
package. All the free-induction decays were subjected to the standard Fourier transformation procedure with line broadening $\left({ }^{13} \mathrm{C}: 50-100 \mathrm{~Hz} ;{ }^{1} \mathrm{H}: 20 \mathrm{~Hz}\right)$ and phasing. The chemical shifts were externally referenced to adamantane $\left({ }^{13} \mathrm{C} \delta: 29.5 \mathrm{ppm} ;{ }^{1} \mathrm{H} \delta: 1.63 \mathrm{ppm}\right)$ secondary to tetramethylsilane $\left({ }^{13} \mathrm{C}\right.$ and ${ }^{1} \mathrm{H}$ $\delta: 0.0 \mathrm{ppm})$.

The proton NMR imaging measurements were performed on the spectrometer described above fitted with a microimaging accessory. The maximum possible amplitude available for the magnetic field gradients was $97.3 \mathrm{G} \mathrm{cm}^{-1}$. Cylindrical monoliths of each sample cut into pieces of about $10 \mathrm{~mm}$ in length were prepared and analyzed by ${ }^{1} \mathrm{H}$ MRI after being dried overnight under vacuum at room temperature (Figure 1). Glass tubes with an outer diameter of $20 \mathrm{~mm}$ and a height of $8 \mathrm{~cm}$ were employed as sample holders. The cylindrical samples were fully impregnated with cyclohexane under vacuum, and loaded into an NMR probe fitted with a radiofrequency coil insert of diameter $25 \mathrm{~mm}$. All measurements were performed at $25 \pm 0.2^{\circ} \mathrm{C}$.

Scout images were recorded using a two-dimensional multislice gradient-echo pulse sequence with an echo time (TE) of $1.86 \mathrm{~ms}$ and a repetition time (TR) of $300 \mathrm{~ms}$. The images were obtained in three orthogonal planes, $1 \mathrm{~mm}$ thick, with a field of view (FOV) of $25 \times 25 \mathrm{~mm}^{2}$ and an isotropic resolution of $128 \times 128$ pixels. To characterize the morphological features of the xerogels, fully relaxed (TR $>5 \times \mathrm{T} 1$ ) multislice/multispin-echo images at echo times between 5 and $40 \mathrm{~ms}$ were recorded. Eight transaxial images with a slice thickness of $0.5 \mathrm{~mm}$, a gap between slices of $0.4 \mathrm{~mm}$, and an in-plane isotropic resolution of $78 \times 78 \mu \mathrm{m}^{2}$ were obtained. $\mathrm{T}_{1}$ measurements were performed using an inversion-recovery 
pulse sequence. The images were processed using ImageJ software (National Institute of Health, Bethesda, US).

\subsubsection{Fourier Transform Infrared Spectroscopy}

The chemical surface characterization of the powdered samples was carried out by Fourier Transform Infrared Spectroscopy (FTIR). Samples were dried overnight before characterization. The spectra were recorded in the 525 and $4000 \mathrm{~cm}^{-1}$ range on a Nicolet IR 8700 spectrometer fitted with a DTGS detector (deuterated triglycine sulphate) at a $4 \mathrm{~cm}^{-1}$ resolution in 64 accumulated scans. The samples were analyzed twice. In order to prepare the tablets, the samples and $\mathrm{KBr}$ (previously dried overnight) were mixed in a proportion of 1:100 in an agate mortar for 10 minutes until a homogeneous mixture was obtained. Around $0.125 \mathrm{~g}$ of this mixture was subjected to 8 tons of pressure in a $13 \mathrm{~mm}$ diameter matrix.

\section{Results and Discussion}

Two samples synthesized under the same conditions, except for the methanol content in the formaldehyde solution (i.e., 0.7 and 12.5), were studied by means of different techniques. The pore size distribution of each sample, both before and after carbonization: $\mathrm{OX}$ and $\mathrm{CX}$ respectively, is shown in Figure 2. It can be seen that a decrease in the methanol content of the formaldehyde solution causes an increase in the pore diameter of up to two orders of magnitude. Moreover, an increase in the methanol content produces an increase in the bulk density of the material from 0.34 to $0.41 \mathrm{~g} / \mathrm{cm}^{3}$ for OX-0.7 and OX-12.5, respectively (Table 1), leading to radically different material. These variations may result from a change of supplier or if a different batch of formaldehyde from the same supplier is used. The differences in methanol content are of crucial importance since the main advantage of organic and carbon gels are that they allow porosities to be tailored for specific applications.

To investigate possible differences in the chemical structure of xerogels, solid state NMR spectroscopy and FTIR measurements were carried out. Figure 3 a shows the ${ }^{13} \mathrm{C} \mathrm{CP} / \mathrm{MAS}$ NMR spectra corresponding to samples OX-0.7 and OX-12.5. The spectra are not significantly different and show 5 main resonances centered at $151.7 ; 132.4 ; 120.5 ; 103.7$, and $28.3 \mathrm{ppm}$. The 151.7 peak corresponds to phenolic carbons from the substituted resorcinol. Due to the fact that aromatic rings can be substituted by one, two or three bridges with respect to the adjacent rings, this gives rise to asymmetric resonances. The peak at 132.4 corresponds to carbons in meta position with respect to both phenols, whereas the peak at $120.5 \mathrm{ppm}$ corresponds to mono and bi-substituted aromatic carbons in ortho position relative to the phenol group. The peak at $103.7 \mathrm{ppm}$ corresponds to the carbons between the phenolic $\mathrm{OH}$ and the wide peak at 28.3 is assigned to different $\mathrm{CH}_{2}$ bridges [22] 
The ${ }^{13} \mathrm{C}$ CP/MAS NMR spectra of samples CX-0.7 and CX-12.5 are shown in Figure 3b. In this case, there is only one peak centered at ca. $127 \mathrm{ppm}$ that can be assigned to $\mathrm{C}-\mathrm{C} \mathrm{sp}^{2}$ bonds (the other peaks in the top trace are spinning sidebands that have not been completely suppressed). As CP/MAS experiments favor the detection of carbons with nearby protons, a comparison of the spectra of samples OX and CX, suggests that the CX samples have a lower proton density than the OX ones, especially, sample CX-12.5 (which has lower signal intensity than the rest). It should also be pointed out that the spectra of the OX samples are the result of averaging 1,000 scans, while those of CX represent an average of 10,000 scans.

Figure 4 shows the ${ }^{1} \mathrm{H}$ MAS spectra of the organic samples (OX) analyzed before and after drying. The spectra in Figure 4a correspond to sample OX-0.7. A narrow peak at 4.62 ppm only appears in the sample prior to drying (top trace), and the broad peak at $3.94 \mathrm{ppm}$ shifts to 3.49 after drying (bottom trace). Likewise, Figure $4 \mathrm{~b}$ illustrates the spectra corresponding to sample OX-12.5. In this case, only one peak at $4.06 \mathrm{ppm}$ is visible before drying. After drying, there is only one broad peak at $3.87 \mathrm{ppm}$ is observed. In both cases, the narrow linewidth of the peaks observed in the xerogels before drying suggests that they are associated to protonated species with a high mobility. The broad peaks observed in the dried samples (bottom traces in Figure 4) could be associated to protons with a low mobility (protons bound to the polymer network). The carbonized samples were also characterized. However, the differences between the pristine and dried samples are very small, reflecting the weak hydrophilic character of the CX samples due to the absence of surface groups (Figures S1 and S2 in the supplementary material).

The FTIR spectra of the OX xerogels are presented in Figure 5. Both spectra exhibit a broad band between 3700 and $3000 \mathrm{~cm}^{-1}$ associated with $\mathrm{O}-\mathrm{H}$ stretching vibrations, due to phenol groups with a high concentration of hydroxyl groups on their surfaces, providing these materials with a high hydrophilicity. The band at $2931 \mathrm{~cm}^{-1}$ can be assigned to aliphatic stretching vibrations $\left(\mathrm{CH}_{2}\right)$. The band located at $1474 \mathrm{~cm}^{-1}$ corresponds to $\mathrm{CH}_{2}$ deformation vibrations and the one at $1613 \mathrm{~cm}^{-1}$ to the aromatic ring stretching vibration $(\mathrm{C}=\mathrm{C})$. Stretching vibration bands at 1217 and $1092 \mathrm{~cm}^{-1}$ indicate the presence of methylene ether bridges C-O-C), although they should be not dominant as they were not detected by NMR [23, 24]. It should also be noted that, as in the case of NMR, FTIR revealed identical bands in both organic samples, indicating similar chemical compositions. Elemental chemical analysis (Table 2) also corroborated the similar composition of the OX samples, independently of the amount of methanol used. In fact, according to the values presented in Table 2 the $\mathrm{C} / \mathrm{H}$ and $\mathrm{C} / \mathrm{O}$ ratio are 1.0 and 2.5, respectively, for OX-0.7; and 1.1 and 2.6, respectively, for OX-12.5. These values are also in good agreement with the theoretical ratios from the proposed mechanisms in the bibliography for RF xerogels 
(i.e. 1.0 and 2.6 for $\mathrm{C} / \mathrm{H}$ and $\mathrm{C} / \mathrm{O}$, respectively). The carbonized samples (CX) were also analyzed, and again their chemical composition seems to be independent of the amount of methanol used (Table 2). The CX samples have a carbon content of ca. $95 \mathrm{wt} \%$. The FTIR spectra of the CX samples reveal a poor surface chemistry, a feature typical of a carbonized material (see Figure S3). It is also worth noting that in no case do these xerogels (OX and CX samples) show signs of impurity content.

It is clear from Figure 4 that all of these organic materials are sensitive to air humidity since water is easily absorbed by their surfaces. Furthermore, Figure 5 shows a high hydroxyl group content on the surface of these organic materials which endows them with a high hydrophilicity. What is more, their porosity provides them with a good water storage capacity, giving rise to a material with outstanding desiccant properties [25]. The FTIR spectra of the CX samples (data not shown) were characteristic of high temperature carbon materials, i.e., there being no bands detected.

As differences in porosity are a result of different reaction mechanisms, and possibly different functional groups in the chemical structure, XPS was also performed as being a more sensitive technique for the characterization of the surface chemistry of the materials. It should be noted that the OX samples could not be characterized by this technique, due to their high volatiles content (i.e. 50 wt $\%$ ) which may cause damage to the XPS equipment.

XPS analyses of the CX carbons brought about a similar atomic composition, namely $89.5 \%$ of $\mathrm{C}$ and $10.5 \%$ of $\mathrm{O}$. As expected, a higher concentration of oxygen is thus present on the outermost layers of the carbon materials when compared to the bulk, as probed by the elemental analysis shown in Table 2 . This higher surface oxygen content helps to depict some slightly differences between the two CX materials, hence on the possible influence of the methanol content on the final properties of the materials. The deconvolution process of the high resolution $\mathrm{C} 1 \mathrm{~s}$ profiles is shown in Figure 6. Four different peaks were considered for the fitting of the original signals (cross symbols). The main peak corresponding to $\mathrm{C}\left(\mathrm{sp}^{2}\right)$ was located at $284.5 \mathrm{eV}$ (B.E.). C-OR (R including $\mathrm{H}$ ) moieties are known to give a peak displaced by approx. $1.5-2 \mathrm{eV}$ of the $\mathrm{C}\left(\mathrm{sp}^{2}\right)$ band. Two additional peaks could be present at +3.5 and $+4.5 \mathrm{eV}$ of the main band, normally assigned to $\mathrm{R}-\mathrm{C}=\mathrm{O}$ and $\mathrm{O}=\mathrm{C}-\mathrm{OR}(\mathrm{R}$ including $\mathrm{H})$ species, respectively. The convolved profile of all these components is also included in Figure 6 (continuous line) to indicate the soundness of the fitting procedure. The relative areas of the different components are collected in Table 3.

It was notice that both samples (CX-12.5 and CX-0.7) present a very similar chemistry. The most relevant difference between them is the relative contribution of $\mathrm{O}=\mathrm{C}-\mathrm{OR}$ functionalities, with a little higher presence in the sample CX-12.5. These would suggest that this carbon material, prepared using 
the formaldehyde solution containing a higher content of $\mathrm{MeOH}$, leads to the formation of a slightly higher number of ester bonds which are most likely related to high levels of crosslinking between the nodules. This would agree with a more condensed structure, leading narrow pores as it is observed in the porosity characterization mentioned above, besides the SEM and TEM observations that would be discussed later.

Figure 7 shows structural differences between the carbonized samples. These differences arise from the differences during polymerization process. However, due to the impossibility to analyze the RF xerogels because of their volatile compounds, only the photographs of the carbonized samples are shown. The SEM photographs reveal significant differences between their internal structures since, on the same scale, large well-defined clusters are recorded for CX-0.7 whereas only small nodules are observed in the case of CX-12.5. TEM also reveals very different patterns, as it is difficult for electrons to penetrate through the sample in the analysis of CX-0.7 due to the large size and thickness of the nodules. Nevertheless this technique is suitable for the characterization of CX-12.5 as its nodules are thinner and homogeneous throughout the sample and therefore, electrons are able to pass through the sample. All of the techniques applied show clearly that these two samples have totally different structures.

Magnetic resonance imaging (MRI) methods allow the non-destructive evaluation of a specimen, providing contrasting images based on its chemical, structural and morphological characteristics [2628]. Moreover, longitudinal studies and a direct comparison between the results obtained by means of MRI and other techniques applied to the same set of samples are also possible.

In this work, the proton MRI study of carbon xerogels impregnated with a fluid (cyclohexane) provides valuable information about their morphological characteristics and could contribute to a better understanding of the effect of the initial concentration of methanol on the synthesis of carbon xerogels. Although MRI is not a very sensitive technique and the degree of spatial resolution does not allow a satisfactory discrimination between submicron structures, it nevertheless enables the open porosity (the porosity in the sample connected to the outside) and, therefore, its regional variations at a macroscopic scale to be mapped [29-31]. Figure 8 shows four representative transaxial proton images corresponding to the four types of synthesized xerogels vacuum impregnated with cyclohexane. In these images, the sample is at the centre, surrounded by cyclohexane. The values of image signal intensity reflected in the different shades of grey are proportional to the amount of cyclohexane protons at each location. Thus, the contrasting shades of each image represent regional variations in the amount of cyclohexane and, in turn, in the open porosity of the sample. The bright rim around the samples corresponds to $100 \%$ cyclohexane. It can be seen that the images corresponding to the xerogels prepared with an initially lower content of methanol $(0.7 \% \mathrm{w} / \mathrm{w})$ have a courser appearance than those prepared with higher 
methanol content $(12.5 \% \mathrm{w} / \mathrm{w})$. This is in agreement with the electron microscopy observations (TEM and SEM) described previously, and with the data in Table 1, since an increase in the methanol content indicates an increase in density, which naturally entails a decrease in porosity.

Different reaction mechanisms are proposed based on a detailed chemical and structural analysis. First, however, it should be mentioned that methanol is added to the formaldehyde solutions in order to prevent the formaldehyde from polymerizing itself and subsequent precipitation. As illustrated in Figure 9, the reaction of methanol with formaldehyde is favored in acidic media, giving rise to mainly hemiacetals (Figure 9a) and to acetals (Figure 9b) if the reaction continues any length of time. The reaction between resorcinol and formaldehyde only takes places if the formaldehyde remains in its free form or in other words, when it is not in a hemiacetal or acetal form. An increase in the methanol content leads to an increase in the hemiacetal form, preventing the resorcinol molecules from finding free formaldehyde molecules to react with.

Furthermore, the greater the amount of free formaldehyde available, the smaller the number of interconnections formed between large-size nodules, as is corroborated by Figures 5-6. On the other hand, the smaller the concentration of free formaldehyde, the larger the number of interconnections formed between the small size nodules. It can also be said that, a similar pattern is observed when there is a reduction in the $\mathrm{R} / \mathrm{F}$ molar ratio [14]. The lower the $\mathrm{R} / \mathrm{F}$ ratio is, the higher the proportion of methanol present since more formaldehyde is consumed in the reaction media which will lead to the effects of the methanol being more prominent.

It is well known that all chemical variables involved in carbon xerogel synthesis are interdependent. However, although the effect of methanol is always apparent, the extent of the effect depends on the other variables. For example, the start of the reaction is favored when the $\mathrm{pH}$ of the initial precursor solution (ca. 3) is increased due to the addition of the basic catalyst. The formation of the resorcinol anions to initiate the addition reaction is favored (see Figure 10). As the kinetics of the reaction increases, small clusters are formed, giving rise to materials with a small pore size. If the kinetics of the reaction is increased, the methanol content is not as important as it would be at a low $\mathrm{pH}$. In this case, crosslinking reactions that counteract the growth of nodules are favored. Consequently the availability of formaldehyde due to methanol content has less influence on the porosity of the material. In short, methanol content has more effect when the kinetics of the reaction is slow or, in other words, when low amount of catalyst is used.

The effect of the dilution ratio has also been widely studied in the literature. However, there are some differences between authors possibly due to the different synthesis methods or drying methods 
employed [13, 32, 33]. Under microwave heating, the sol-gel reaction proceeds faster than under conventional heating, leaving the solvent no time to evaporate and so it is retained inside the structure and this may alter the structure somewhat. A lower dilution degree implies a higher proportion of reactants in the solution and, therefore, an increase in the number of smaller size nodules formed. On the other hand, an increase in the dilution ratio will cause the formation of a smaller number of nodules of larger size. Furthermore, a high dilution ratio will lead to a lower degree of crosslinking and materials with poorer mechanical properties. If the dilution ratio is increased even further, gel will not be able to form since the nodules will be too far apart to crosslink [34].

\section{Conclusions}

The concentration of methanol present in commercial formaldehyde solutions has a significant influence on the porosity of RF xerogels. The bulk chemical composition remains analogous in spite of some differences in the structure and possibly some functional groups. Different reaction mechanisms have been proposed to explain the strong dependence of the final structure of RF xerogels on the presence of methanol. Methanol reacts with formaldehyde, generating hemiacetal molecules which are not able to react with resorcinol. Therefore, the greater the amount of methanol, the smaller the amount of formaldehyde that will be available to participate in reactions, which means that resorcinol molecules will have difficulty finding formaldehyde in its free form to react with. In short, organic and carbon xerogels synthesized with a lower concentration of methanol show a higher level of porosity with larger pore sizes (by up to two orders of magnitude) than when formaldehyde with high concentrations of methanol is used. Consequently, in order to obtain accurate bespoke materials, apart from the already established chemical variables (i.e., $\mathrm{pH}$ of the precursor solution, $\mathrm{R} / \mathrm{F}$ ratio, dilution ratio, etc) it is necessary to take into account the composition of the formaldehyde solution and especially the percentage of methanol.

\section{Acknowledgements}

The authors gratefully acknowledge the financial support of the Ministerio de Economía y Competitividad of Spain, MINECO (Projects CTQ2014-54772-P). 


\section{References}

1. Pekala R (1989) Organic aerogels from the polycondensation of resorcinol with formaldehyde. $J$. Mater. Sci. 24:3221-3227.

2. Job N et al (2006) Synthesis optimization of organic xerogels produced from convective airdrying of resorcinol-formaldehyde gels. J. Non-Cryst. Solids 352:24-34.

3. Léonard A et al (2008) Evolution of mechanical properties and final textural properties of resorcinol-formaldehyde xerogels during ambient air drying. J. Non-Cryst. Solids 354:831-838.

4. Shen J, Hou J et al (2005) Microstructure control of RF and carbon aerogels prepared by sol-gel process. J. Sol-Gel Science and Technology 36:131-136.

5. Feng J, Feng J, Zhang C (2011) Shrinkage and pore structure in preparation of carbon aerogels. J. Sol-Gel Science and Technology 59:371-380.

6. Pérez-Caballero F et al (2008) Preparation of carbon aerogels from 5-methylresorcinolformaldehyde gels. Microporous Mesoporous Mater. 108:230-236.

7. Szczurek A et al (2011) The use of tannin to prepare carbon gels. Part II. Carbon cryogels. Carbon 49:2785-2794.

8. Rey-Raap N et al (2015) Towards a feasible and scalable production of bio-xerogels. J. Colloid Interface Sci. 456:138-144.

9. Moreno-Castilla C, et al (2011) Surface characteristics and electrochemical capacitances of carbon aerogels obtained from resorcinol and pyrocatechol using boric and oxalic acids as polymerization catalysts. Carbon 49:3808-3819.

10. Zubizarreta L, Arenillas A, Pis JJ (2008) Preparation of Ni-doped carbon nanospheres with different surface chemistry and controlled pore structure. Applied Surface Science 254:3993-4000.

11. Szczurek A et al (2016) Structure and properties of poly(furfuryl alcohol)-tannin polyHIPEs. Eur. Polymer Journal. 78:195-212.

12. Rey-Raap N, Menéndez JA, Arenillas A (2014) Optimization of the process variables in the microwave-induced synthesis of carbon xerogels. J. Sol-Gel Sci. Technol. 69:488-497.

13. Rey-Raap N, Menéndez JA, Arenillas A (2014) RF xerogels with tailored porosity over the entire nanoscale. Microporous Mesoporous Mater. 195:266-275. 
14. Rey-Raap N, Menéndez JA, Arenillas A. (2014) Simultaneous adjustment of the main chemical variables to fine-tune the porosity of carbon xerogels. Carbon 78:490-499.

15. Moreno AH et al (2013) Carbonisation of resorcinol-formaldehyde organic xerogels: Effect of temperature, particle size and heating rate on the porosity of carbon xerogels. J. Anal. Appl. Pyrol. 100:111-116.

16. Al-Muhtaseb SA, Ritter JA (2003) Preparation and Properties of Resorcinol-Formaldehyde Organic and Carbon Gels. Advanced Materials. 15:101-114.

17. Alonso-Buenaposada ID et al (2015) Effect of methanol content in commercial formaldehyde solutions on the porosity of RF carbon xerogels. J. Non-Cryst. Solids. 426:13-18.

18. Calvo EG et al (2011) Fast microwave-assisted synthesis of tailored mesoporous carbon xerogels. J. Colloid Interface Sci. 357:541-547.

19. Singh B, Hesse R, Linford MR (2015) Good practices for XPS (and other types of) peak fitting. Vacuum Tecnology \& Coating 12:25-31.

20. Figueiredo JL, Pereira (2010) MFR The role of surface chemistry in catalysis with carbons. Catalysis Today 150:2-7.

21. Estrade-Szwarckopf H (2004) XPS photoemission in carbonaceous materials: a "defect" peak beside the graphitic asymmetric peak. Carbon 42:1713-1721.

22. Mulik S, Sotiriou-Leventis C, Leventis N (2007) Time-Efficient Acid-Catalyzed Synthesis of Resorcinol- Formaldehyde Aerogels. Chem. Mater. 19:6138-6144.

23. Wickenheisser $\mathrm{M}$ et al (2015) Hierarchical MOF-xerogel monolith composites from embedding MIL-100(Fe,Cr) and MIL-101(Cr) in resorcinol-formaldehyde xerogels for water adsorption applications. Microporous Mesoporous Mater. 215:143-153.

24. Awadallah-F A, Elkhatat AM, Al-Muhtaseb SA (2011) Impact of synthesis conditions on mesoand macropore structures of resorcinol-formaldehyde xerogels. J. Mater. Sci. 46:7760-7769.

25. Alonso-Buenaposada ID et al (2016) Desiccant capability of organic xerogels: surface chemistry vs porous texture. Microporous Mesoporous Mater. 232:70-76.

26. Blümich B, Callaghan PT (1995) In Principles of nuclear magnetic resonance microscopy. Oxford University Press, Oxford, Magnetic Resonance in Chemistry 33:322-322.

27. Blumich B In NMR imaging of materials. OUP Oxford. Vol. 2000, 57. 
28. Stapf S, Han SI (2006) In NMR imaging in chemical engineering. John Wiley \& Sons.

29. Ackerman JL et al (1987) In The use of NMR imaging to measure porosity and binder distributions in green-state and partially sintered ceramics. Massachusetts General Hospital, Boston (USA). Radiology Dept.; Argonne National Lab., IL (USA); Aluminum Co. of America, Alcoa Center, PA. Alcoa Technical Center.

30. Garrido L (1999) Nondestructive evaluation of biodegradable porous matrices for tissue engineering. Tissue engineering methods and protocols 35-45.

31. Marcos $\mathrm{M}$ et al (2006) NMR relaxometry and imaging of water absorbed in biodegradable polymer scaffolds. Magnetic resonance imaging 24:89-95.

32. Job N et al (2005) Carbon aerogels, cryogels and xerogels: Influence of the drying method on the textural properties of porous carbon materials. Carbon 43:2481-2494.

33. Tamon H et al (1997) Porous structure of organic and carbon aerogels synthesized by sol-gel polycondensation of resorcinol with formaldehyde. Carbon 35:791-796.

34. Rey-Raap N, Arenillas A, Menéndez JA (2015) Formaldehyde in the synthesis of resorcinolformaldehyde carbon gels. In Formaldehyde: Synthesis, applications and potential health effects. Patton A. Ed.; Nova Science Publishers.; pp 31-60. 
Table 1 . Textural properties of the samples obtained by mercury porosimetry

\begin{tabular}{cccc}
\hline Sample & $\begin{array}{c}\text { Pore volume } \\
\left(\mathbf{c m}^{\mathbf{3}} \mathbf{g}^{-\mathbf{1}}\right)\end{array}$ & $\begin{array}{c}\text { Bulk density } \\
\left(\mathbf{g ~ c m}^{\mathbf{3}}\right)\end{array}$ & \% Porosity \\
\hline OX-0.7 & 2.02 & 0.34 & 69 \\
\hline $\mathrm{OX}-12.5$ & 1.61 & 0.41 & 66 \\
\hline $\mathrm{CX}-0.7$ & 2.13 & 0.32 & 69 \\
\hline $\mathrm{CX}-12.5$ & 1.53 & 0.43 & 65 \\
\hline
\end{tabular}


Table 2. Elemental analysis results for the organic and carbon xerogels (dry basis)

\begin{tabular}{lcccc}
\hline & OX-0.7 & OX-12.5 & CX-0.7 & CX-12.5 \\
\hline $\mathrm{C}( \pm 0.2$, wt. \%) & 62.5 & 63.2 & 93.7 & 93.4 \\
$\mathrm{H}( \pm 0.2$, wt. \%) & 5.0 & 4.8 & 1.8 & 1.7 \\
$\mathrm{~N}( \pm 0.2$, wt. \%) & 0.3 & 0.3 & 0.5 & 0.6 \\
$\mathrm{O}( \pm 0.2$, wt. \%) & 32.2 & 31.7 & 4.0 & 4.3 \\
\hline
\end{tabular}


Table 3. Analysis of the C1s high resolution XPS of the carbonised gels

\begin{tabular}{lcccc}
\hline \multirow{2}{*}{ Sample } & \multicolumn{5}{c}{$\begin{array}{c}\text { Relative contribution of the fitting } \\
\text { peaks (\%) }\end{array}$} \\
\cline { 2 - 5 } CX-0.7 & C=C & C-OR & RC=O & O=C-OR \\
\hline CX-12.5 & 68.2 & 20.4 & 4.4 & 7.0 \\
\hline
\end{tabular}




\section{FIGURE CAPTIONS}

Figure 1. Samples of the xerogels prepared for the MRI study.

Figure 2. Pore size distribution obtained by mercury porosimetry for the samples studied.

Figure $3 .{ }^{13} \mathrm{C} \mathrm{CP} / \mathrm{MAS}$ NMR spectra with proton decoupling and sideband suppression corresponding to the samples studied: a) organic xerogels and b) carbon xerogels. Asterisks indicate residual spinning side bands and the structures assigned to the peaks appear in the inset.

Figure 4. ${ }^{1} \mathrm{H}$ MAS NMR spectra corresponding to a) OX-0.7 and b) OX-12.5 samples, before (top trace) and after (bottom trace) drying.

Figure 5. Chemical characterization of the organic samples from FTIR spectra.

Figure 6. C1s high resolution XPS spectra of CX-0.7 (a) and CX-12.5 (b).

Figure 7. Microphotograhs of the carbon xerogels obtained from SEM and TEM characterization.

Figure 8. Proton MRI of xerogels vacuum impregnated with cyclohexane. Images acquired with an echo time of $5 \mathrm{~ms}$ and a repetition rate of $6 \mathrm{~s}$. The images represent transaxial cross-sections of the cylindrical xerogel samples.

Figure 9. Reaction mechanisms proposed for the stabilization of formaldehyde by the addition of methanol and the formation of hemiacetals (a) and acetals (b).

Figure 10. Polymerization steps of resorcinol and formaldehyde with basic catalyst. 


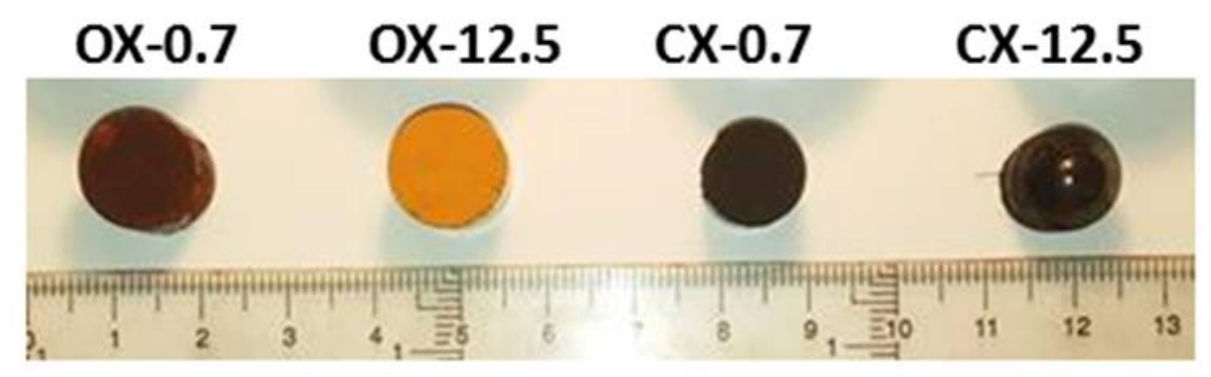

Figure 1 
Pore Size Distribution

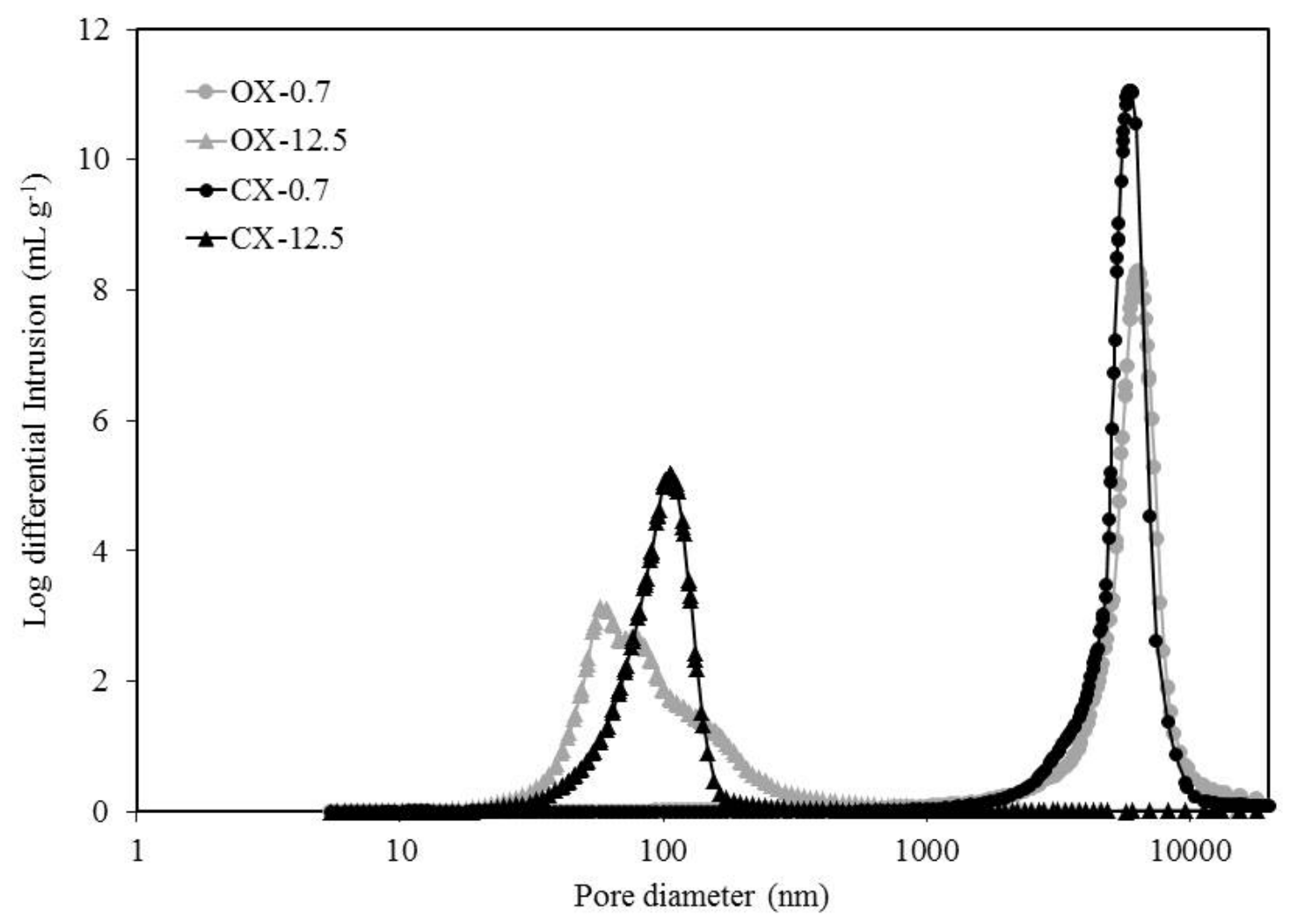

Figure 2 

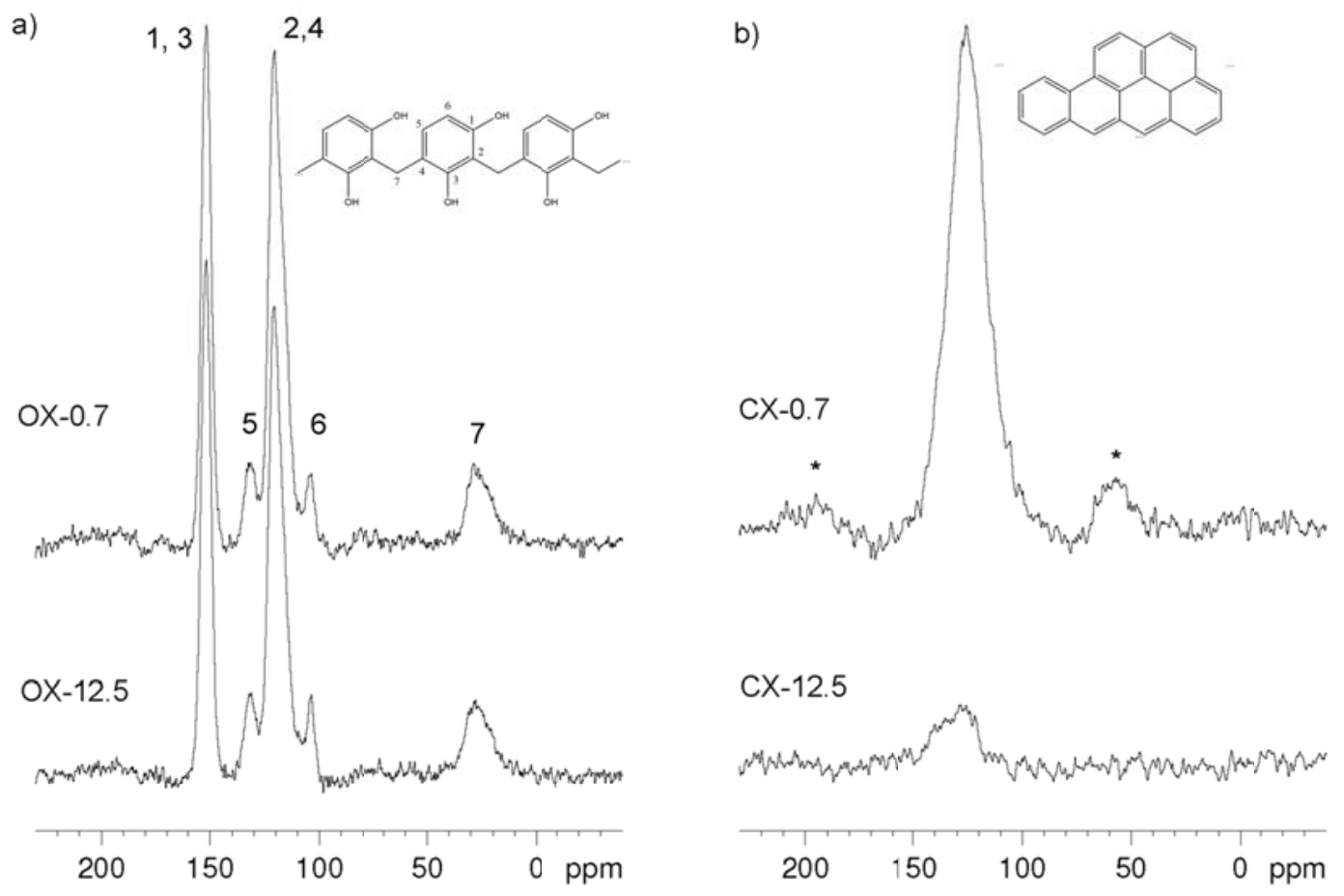

Figure 3 

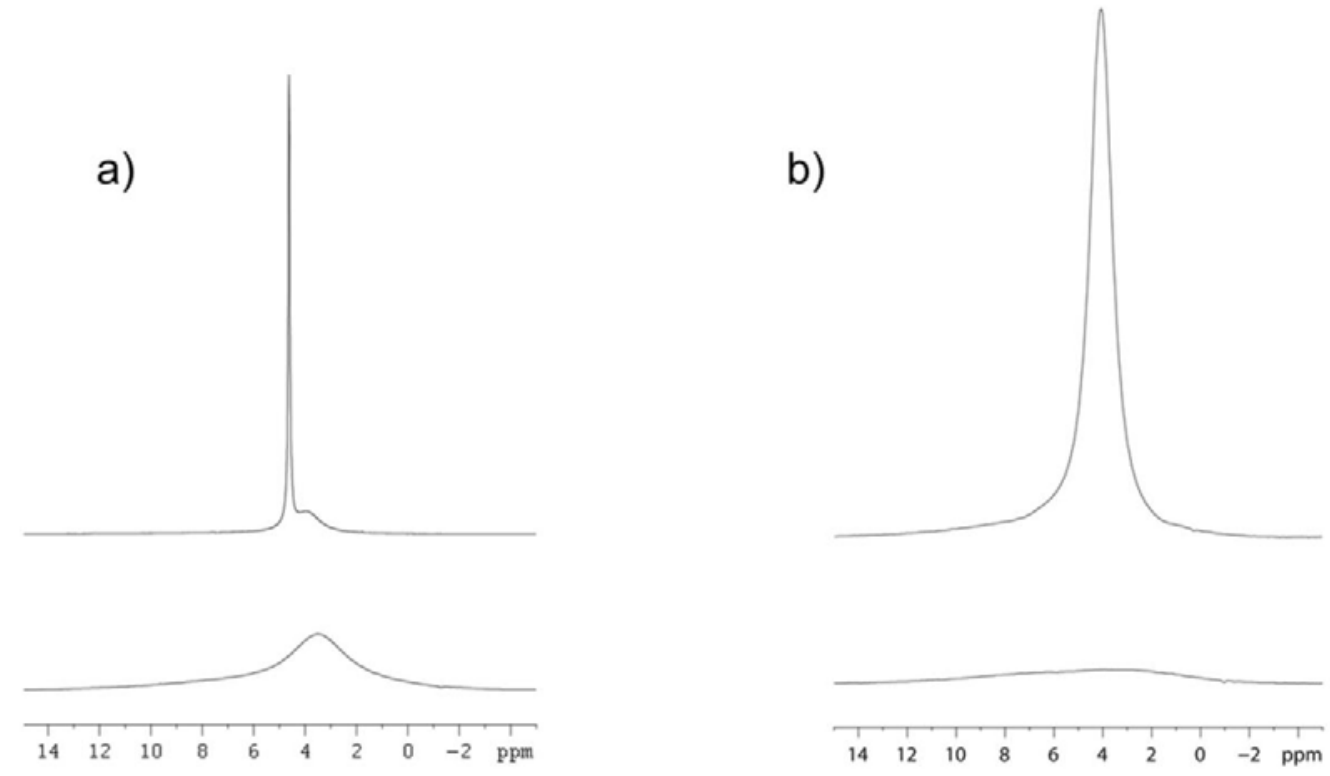

Figure 4 


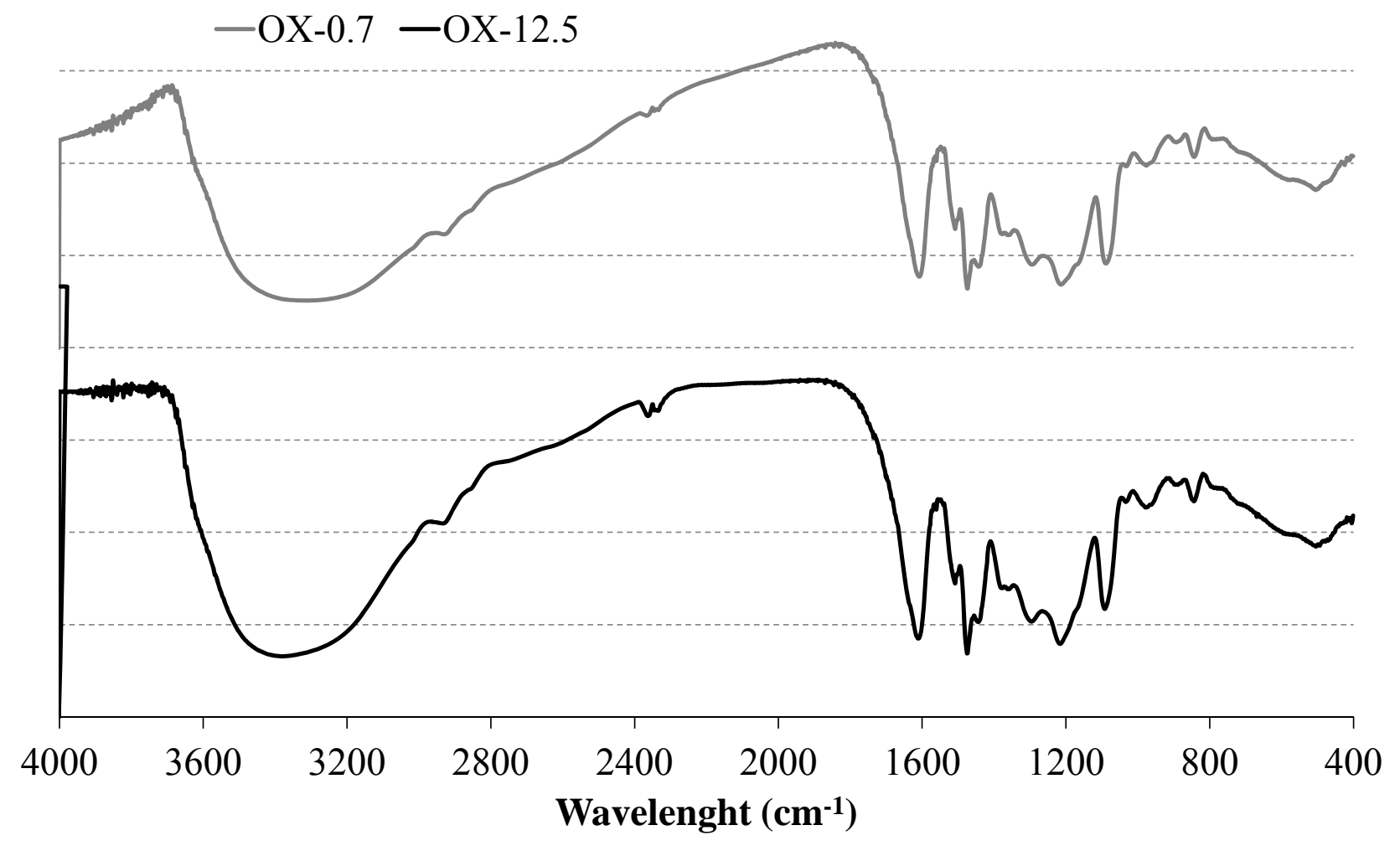

Figure 5 
a)

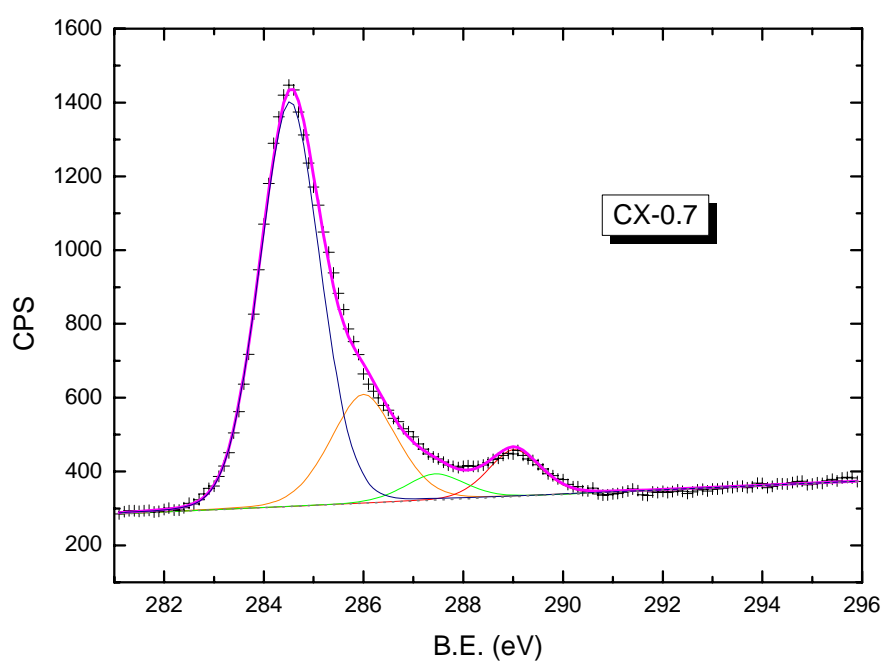

b)

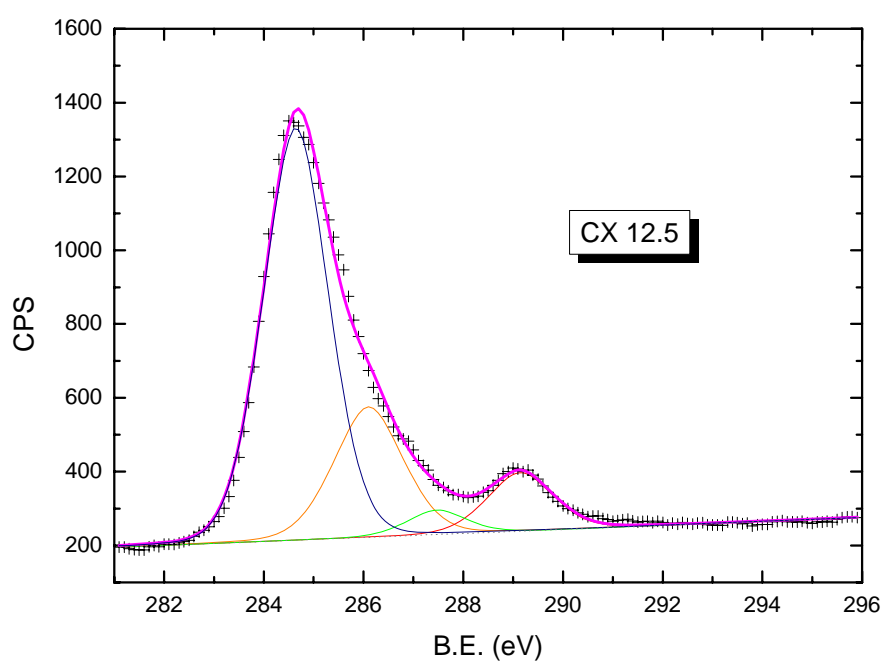

Figure 6 


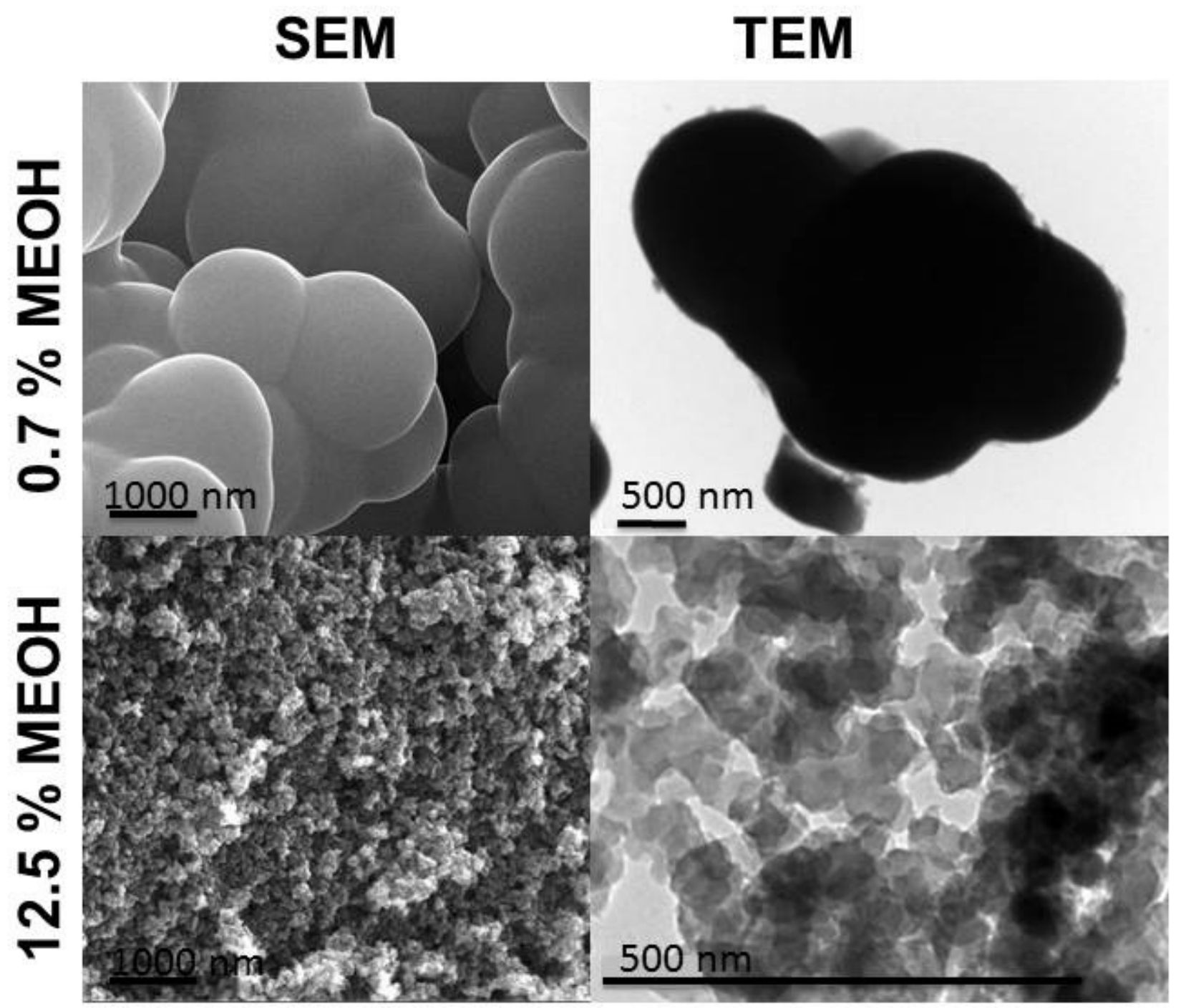

Figure 7 


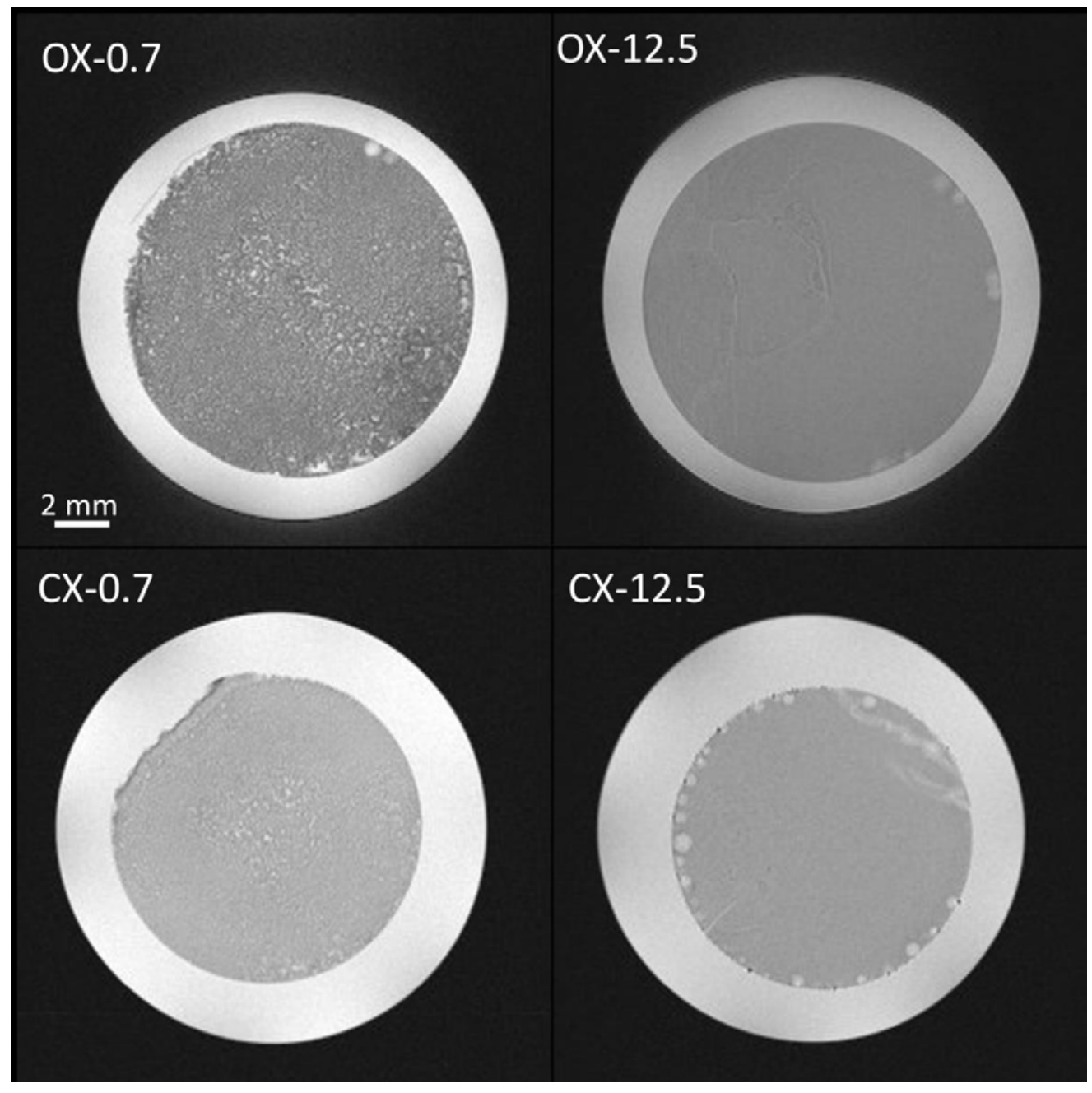

Figure 8 
a)

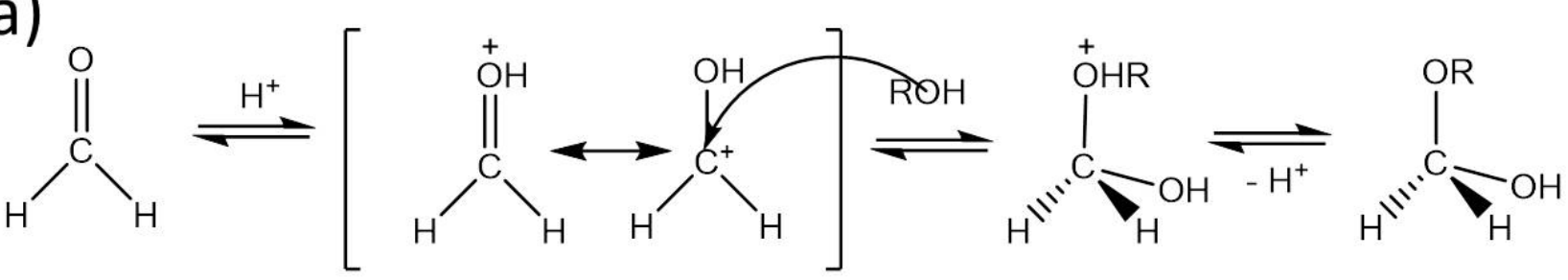

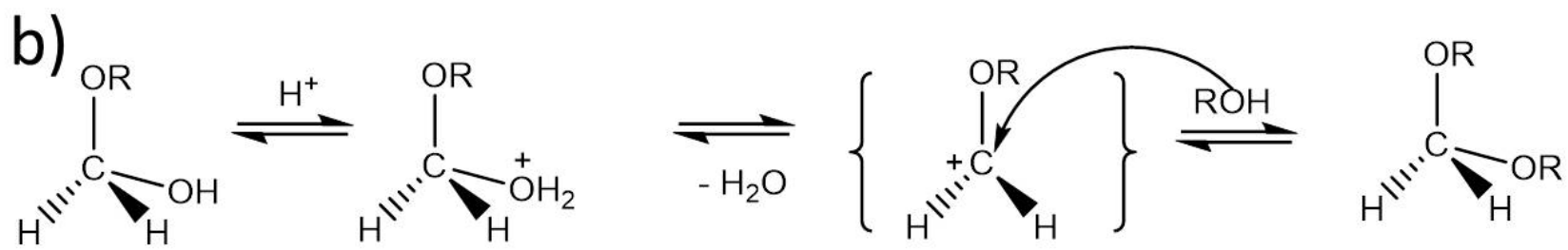

Figure 9 


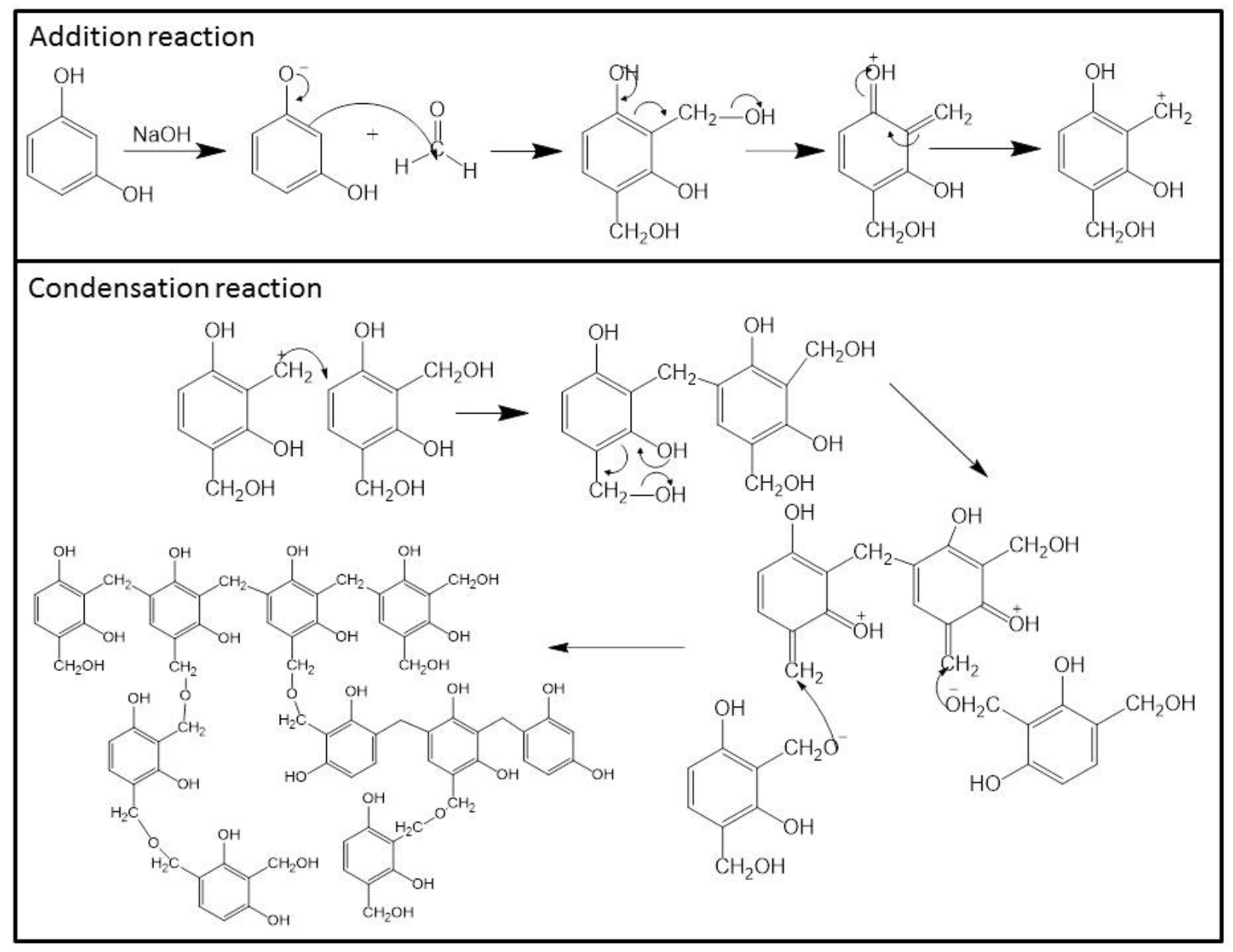

Figure 10 


\section{An underrated variable essential for tailoring the structure of xerogel: the methanol content of commercial formaldehyde solutions}

Isabel D. Alonso-Buenaposada ${ }^{1}$, Leoncio Garrido ${ }^{2}$, M.A. Montes-Morán ${ }^{1}, J_{\text {. Angel Menéndez }}{ }^{1}$, Ana Arenillas $^{1 *}$

${ }^{1}$ Instituto Nacional del Carbón, CSIC, Apartado 73, 33080 Oviedo, Spain

${ }^{2}$ Departamento de Química Física, Instituto de Ciencia y Tecnología de Polímeros, ICTP-CSIC, Juan de la Cierva 3, 28006 Madrid, Spain

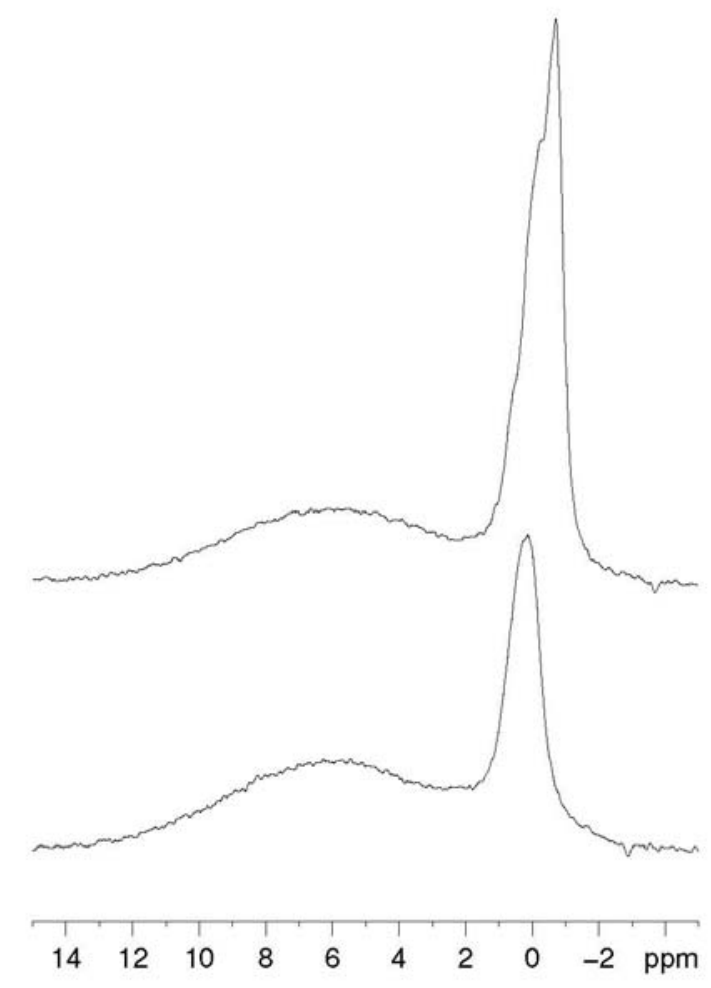

Figure S1. ${ }^{1} \mathrm{H}$ MAS spectra corresponding to sample CX-0.7 before (top trace) and after (bottom trace) drying. The spectra were acquired with the same conditions and are plotted with the same vertical scale. A broad peak centered at about $6 \mathrm{ppm}$ is observed in both spectra. The narrow peak shows changes after 
drying, the peak at $0.13 \mathrm{ppm}$ is observed in the two spectra, while the peak at $-0.71 \mathrm{ppm}$ is only visible in the untreated sample.

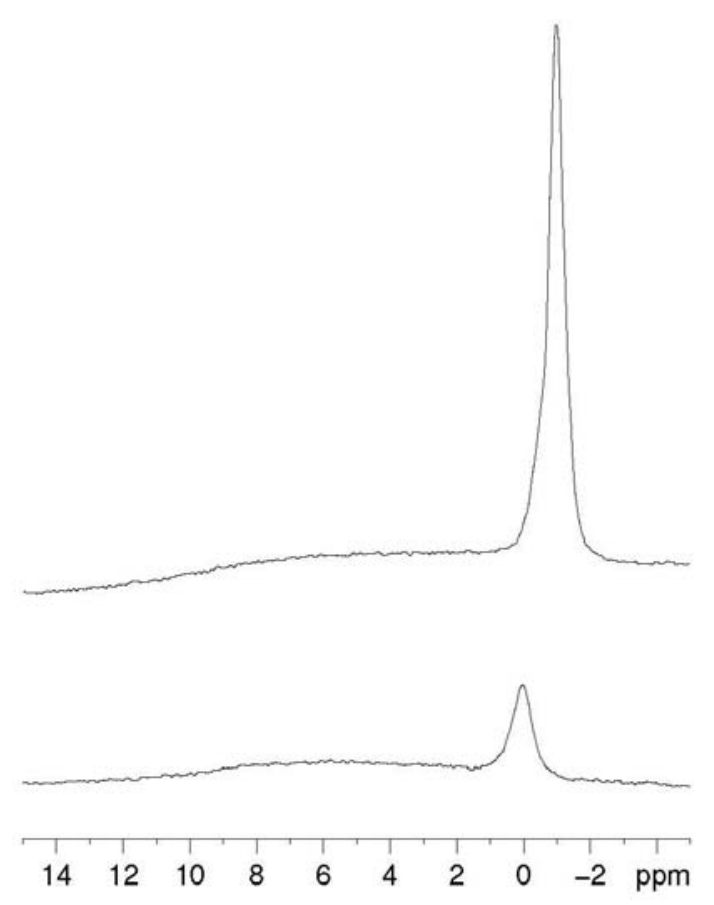

Figure S2. ${ }^{1} \mathrm{H}$ MAS spectra corresponding to sample CX-12.5 before (top trace) and after (bottom trace) drying. The spectra were acquired with the same conditions and are plotted with the same vertical scale. A broad peak centered at about $5.5 \mathrm{ppm}$ is observed in both spectra. The intensity of the narrow peak decreases after drying, and shifts from -0.99 to $0.02 \mathrm{ppm}$. 


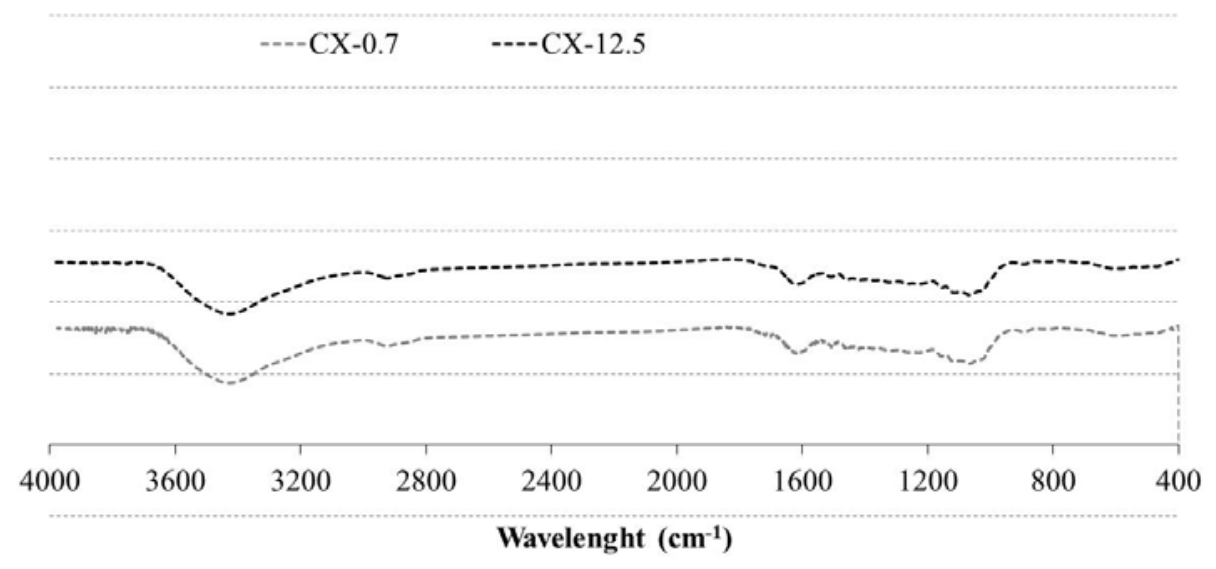

Figure S3. FTIR spectra for the carbonized samples CX-0.7 and CX-12.5 (note, it was used the same scale as Figure 5 for comparison proposes). 